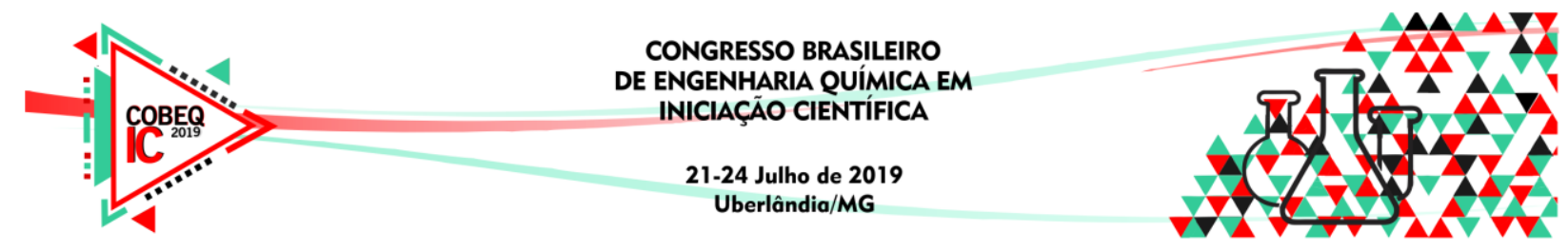

\title{
DETERMINAÇÃO ELETROANALÍTICA DE SELENITO EM ALIMENTOS IN NATURA: BATATA, LARANJA, ESPINAFRE, MAÇÃ E TOMATE
}

\author{
F. C. O. L. MARTINS ${ }^{1}$, J. F. S. NERY ${ }^{1}$ e D. De SOUZA $^{1}$ \\ ${ }^{1}$ Universidade Federal de Uberlândia, Campus de Patos de Minas \\ E-mail para contato: fernandacolm@ hotmail.com
}

\begin{abstract}
RESUMO - O selênio é um micronutriente muito importante para a manutenção do corpo humano, atuante em algumas funções vitais, entretanto a diferença entre a concentração indicativa de deficiência, de essencialidade e de toxicidade é muito pequena. Assim, o objetivo deste trabalho foi o desenvolvimento de uma metodologia capaz de determinar baixas concentrações de selênio presentes em amostras de alimentos in natura usando eletrodo de amálgama de prata modificado com filme de mercúrio (m-AgSAE) aliado à técnica de voltametria de onda quadrada (SWV). Após a otimização experimental (potenciais e tempos de preconcentração) e voltamétrica (frequência de aplicação de pulsos de potencial de $90 \mathrm{~s}^{-1}$, amplitude de aplicação de pulsos de - $25 \mathrm{mV}$ e incremento de varredura de potenciais de $-2 \mathrm{mV}$ ) foram construídas curvas de calibração para avaliação das figuras de mérito da metodologia proposta, tais como sensibilidade, reprodutibilidade, precisão, exatidão e faixa dinâmica linear. A sensibilidade calculada foi de $2,958 \mathrm{ng} \mathrm{L}^{-1}$ e $8,964 \mathrm{ng} \mathrm{L}^{-1}$, para os valores de limite de detecção e quantificação, respectivamente, com coeficiente de correlação de 0,9989 e eficiência de recuperação calculada de 101,00 - 101,10\%, reprodutibilidade de $4,90 \%$ e repetibilidade de $1,64 \%$. A metodologia proposta foi aplicada para a quantificação de selênio em amostras de alimentos in natura tais como maçã, laranja, batata, espinafre e tomate obtendo-se os valores de $6,70 \mu \mathrm{g} \mathrm{L}^{-1}, 5,10 \mu \mathrm{g} \mathrm{L}$ $1,16,17 \mu \mathrm{g} \mathrm{L}^{-1}, 2,53 \mu \mathrm{g} \mathrm{L}^{-1}$ e $38,45 \mu \mathrm{g} \mathrm{L}^{-1}$, respectivamente.
\end{abstract}

\section{INTRODUÇÃO}

Para o perfeito funcionamento do corpo humano é necessário a ingestão de nutrientes e micronutrientes essenciais que atuam na manutenção do metabolismo, as quais auxiliam em reações químicas vitais e/ou como precursores de outras reações importantes. Os minerais são micronutrientes indispensáveis para o funcionamento do corpo humano, dentre eles destacamse o ferro, magnésio, sódio, potássio, cálcio, zinco, iodo, fósforo e selênio, sendo que cada um destes micronutrientes tem um nível nutricional específico (Martins, et al. (2017)). O selênio (Se) é um metaloíde que auxilia no retardamento do envelhecimento, no tratamento de doenças cardiovasculares e neoplasias, distrofia muscular, esclerose múltipla, osteoporose e reprodutibilidade, além disso, possui funções no metabolismo da tireoide, ácidos graxos e vitamina $\mathrm{E}$ ( $\alpha$-tocoferol). Entretanto, a diferença entre a concentração essencial, de deficiência e de toxicidade é muito pequena, e as quantidades ingeridas dependem diretamente da concentração encontrada nos alimentos. A toxidade deste composto pode promover queda de 


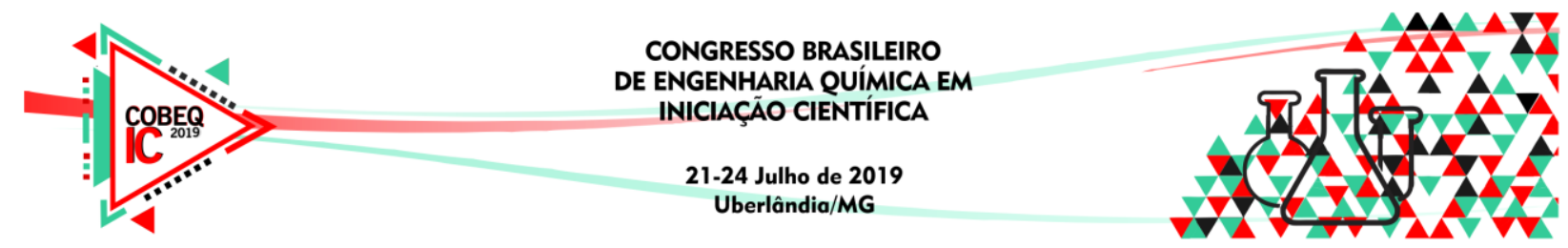

cabelo, unhas quebradiças, irritação na pele e nos olhos, e em alguns casos levar ao óbito (Martins, et al. (2017)).

Os níveis nutricionais e tóxicos devem ser avaliados e para isto, são necessários métodos analíticos capazes de quantificar baixas concentrações para a determinação da quantidade de Se nos alimentos, e altas concentrações para se avaliar níveis tóxicos e de deficiência. Para isto são empregadas técnicas espectroscópicas (as quais empregam medidas de radiação emitida ou absorvida pelas substâncias), técnicas cromatográficas (as quais promovem uma separação de compostos orgânicos ou inorgânicos de acordo com a interação entre a fase móvel e fase estacionária) e técnicas eletroanalíticas (nas quais se mede uma propriedade elétrica relacionada com reações eletroquímicas que acontecem na superfície do eletrodo de trabalho de acordo com a concentração do analito e ainda permite a especiação química do composto de interesse) (Martins, et al. (2017)).

Assim, o objetivo deste trabalho foi o desenvolvimento de uma metodologia eletroanalítica para a determinação de selênio em amostras de alimentos in natura, empregando-se um eletrodo de amálgama de prata modificado com filme de mercúrio (mAgSAE) aliado com a técnica de voltametria de onda quadrada com etapa de preconcentração adsortiva (CS-SWV).

\section{EXPERIMENTAL}

Para os experimentos voltamétricos foi empregada uma célula eletroquímica construída em vidro e com volume aproximado de $10 \mathrm{~mL}$, juntamente com tampa de teflon ${ }^{\circledR}$ para fechar o sistema e dar suporte aos três eletrodos. O eletrodo de trabalho utilizado foi o m-AgSAE, o eletrodo de referência $\left(\mathrm{AgCl} / \mathrm{Cl}^{-1}\right)$, e o eletrodo auxiliar um fio de platina, que tem finalidade de fechar o circuito elétrico. Todos os eletrodos foram construídos no próprio laboratório, e após a montagem da célula eletroquímica foram conectados ao instrumento de medida, onde ocorreu a aplicação dos potenciais elétricos e as respectivas medidas de corrente.

O eletrodo de amálgama foi preparado de acordo com trabalhos prévios, empregando-se a proporção em massa de prata e mercúrio (30/70, $\mathrm{Ag} / \mathrm{Hg} ; \mathrm{m} / \mathrm{m})(\mathrm{DE}$ SOUZA et al. (2010)). Um tratamento eletroquímico para diminuição da resistência à transferência de carga foi realizado empregando-se uma solução de $0,2 \mathrm{~mol} \mathrm{~L}^{-1}$ de $\mathrm{KCl}$ e aplicação de $-2,2 \mathrm{~V}$ por 600 segundos. Após isto, o eletrodo foi colocado em contato com mercúrio líquido durante 30 minutos para a formação do m-AgSAE. O eletrodo de referência empregado foi preparado utilizando-se um filme de cloreto $\left(\mathrm{AgCl} / \mathrm{Cl}^{-1}\right)$ eletroquimicamente depositado na superfície um fio de prata, o qual garante o potencial elétrico do circuito durante as análises.

Todos os reagentes empregados apresentaram alta pureza analítica. As soluções empregadas nos experimentos, tais como soluções de ácido selenoso, ácido clorídrico e cloreto de potássio, foram preparadas com água purificada com um Sistema Purificador de água Osmose Reversa OS50 LX, e com reagentes de alto padrão analítico, balança analítica classe I (Bel Engineering, M214A) com precisão de 0,0001g, vidrarias comuns que foram previamente lavadas com água, sabão neutro e solução $10 \%$ de ácido nítrico.

O equipamento utilizado nas análises foi um potenciostato/ galvanostato microAutolab III Metrohn-Pensalab, o qual foi controlado pelo software NOVA® 2.0, também da Metrohn. 


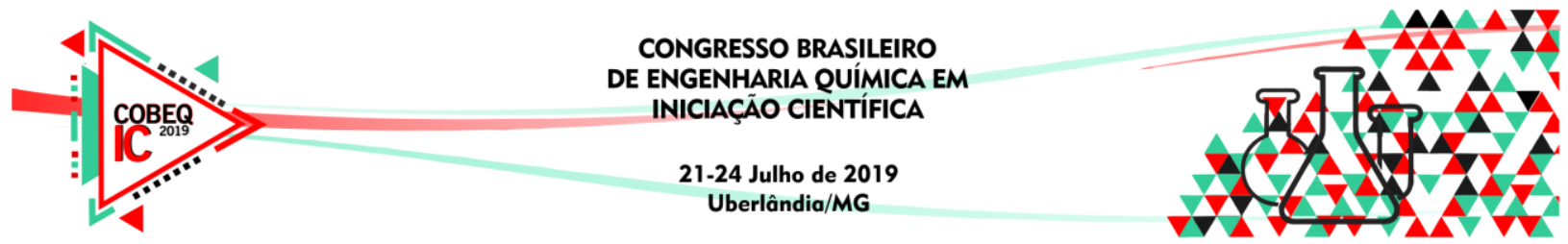

Antes de iniciar as análises, na célula eletroquímica contendo $10 \mathrm{~mL}$ de eletrólito borbulhouse o gás nitrogênio $\left(\mathrm{N}_{2}\right)$ ultrapuro White Martins, para que fosse possível a remoção total do oxigênio dissolvido no meio. No início de cada dia de análises, ou após pausas maiores que uma hora, foi realizado o condicionamento do m-AgSAE em meio de $0,2 \mathrm{~mol} \mathrm{~L}^{-1}$ de $\mathrm{KCl}$ e utilização de experimentos de voltametria cíclica, aplicando um potencial fixo de $-2,20 \mathrm{~V}$ por um tempo de $30 \mathrm{~s}$, seguido de varredura de potenciais de 0,0 a $-2,2 \mathrm{~V}$ com velocidade de varredura de $100 \mathrm{mV} \mathrm{s}^{-1}$.

Para os experimentos voltamétricos foi empregada a CS-SWV, onde ocorre uma etapa de transferência de massa do analito da solução para a superfície do eletrodo promovendo a preconcentração inicial, seguida da etapa de medição, proporcionando-se assim uma diminuição nos limites de detecção e de quantificação. Assim, a SWV baseia-se na aplicação de um pulso de potencial e a corrente resultante é formada de acordo com a sobreposição de uma escada de potencial de altura $a$ com incremento $\Delta \mathrm{E}_{\mathrm{s}}$ e a frequência $1 / \mathrm{t}$, onde t é o tempo de aplicação dos pulsos de potenciais. A medida da corrente é realizada antes e depois da aplicação do pulso, onde a corrente capacitiva é negligenciada, a qual faz com que a SWV tenha uma maior sensibilidade nas quantificações dos analitos e com maior rapidez nas análises (DE SOUZA et al. (2004)).

As amostras foram obtidas no mercado de Patos de Minas, as quais foram previamente digeridas usando ácido nítrico, ácido perclórico e ácido clorídrico de acordo com a metodologia de Inam et al. (INAM (1999). Curvas de recuperação empregando-se método de adição de padrão foram empregadas para avaliar as concentrações de Se em cada uma das amostras. Também foram avaliados a precisão e exatidão da metodologia proposta em cada uma das amostras.

\section{RESULTADOS E DISCUSSÃO}

Testes preliminares foram realizados empregando-se o eletrodo de amálgama de prata (p-AgSAE) e o eletrodo de amálgama de prata modificado com filme de mercúrio (mAgSAE), ambos em meio de uma solução de $0,10 \mathrm{~mol} \mathrm{~L}^{-1}$ de $\mathrm{HCl}$, utilizada como eletrólito suporte. Nestas condições obteve-se respostas diferentes para cada um dos eletrodos, onde foi possível observar a presença de um pico de redução bem definido com potencial de pico $\left(\mathrm{E}_{\mathrm{p}}\right)$ em - 0,64 V sobre o p-AgSAE e -0,50 V sobre o m-AgSAE. Além disso, observou-se que os resultados obtidos sobre o $\mathrm{m}-\mathrm{AgSAE}$ foram mais reprodutíveis, repetíveis e mais seletivos que aqueles obtidos sobre o p-AgSAE, por isto o m-AgSAE foi o escolhido para as etapas posteriores. Os parâmetros de preconcentração, aplicação de potenciais e tempos de preconcentração, para a avaliação do melhor sinal analítico considerando-se a largura do pico voltamétrico e a intensidade do sinal, foram avaliados, observando-se que a aplicação de 0,0 $\mathrm{V}$ durante 45 segundos proporcionou o melhor sinal analítico, como pode ser visualizado na Figura 1.

Os parâmetros da SWV foram avaliados para obtenção de melhor seletividade e sensibilidade. Assim a frequência de aplicação dos pulsos de potenciais $(f)$ foi variada de 10 a $300 \mathrm{~s}^{-1}$, onde observou-se que o aumento do sinal analítico foi proporcional ao aumento de $f$, acompanhado de aumento na largura do pico, diminuindo-se a seletividade. O incremento de varredura de potenciais $(\Delta E s)$ foi variado de - 2 a $-10 \mathrm{mV}$, observando-se que o sinal 


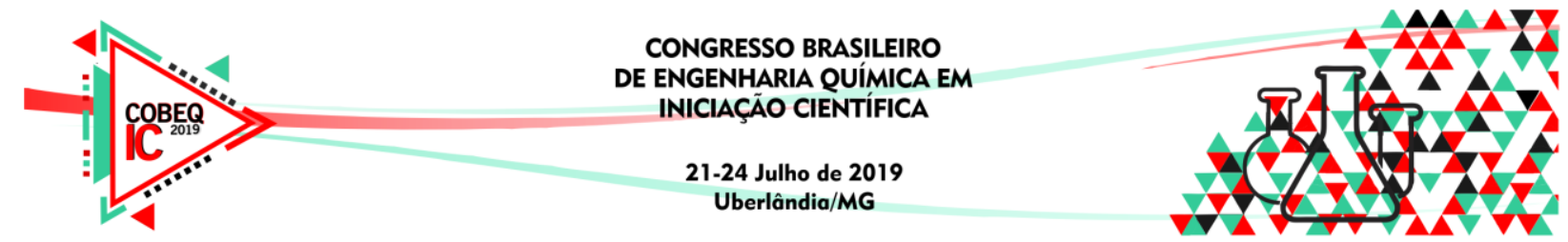

analítico aumentou proporcionalmente com o aumento de $\Delta \mathrm{Es}$, acompanhado de perda no perfil dos picos voltamétricos. A amplitude dos pulsos (a) foi variada de 5 a $50 \mathrm{mV}$, porém observou-se um alargamento do pico e deslocamento do potencial, como pode ser observado nos voltamogramas da Figura 2.

Figura 1 - Voltamogramas de onda quadrada para a redução de $1,00 \times 10^{-6} \mathrm{~mol} \mathrm{~L}^{-1}$ de selenito sobre m-AgSAE empregando-se $f=100 \mathrm{~s}^{-1}, \Delta \mathrm{E}_{\mathrm{s}}=-2 \mathrm{mV}$ e $a=50 \mathrm{mV}$, considerando-se diferentes potenciais de preconcentração durante 30s (a) e variação do tempo de preconcentração (b) .
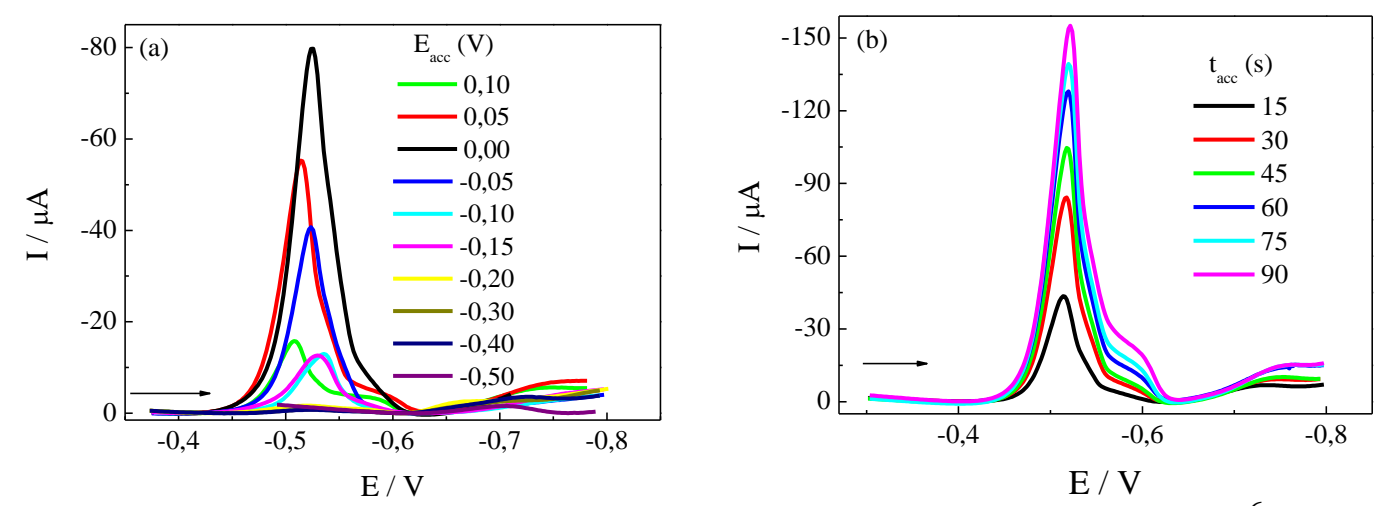

Figura 2 - Voltamogramas de onda quadrada para a redução de $1,00 \times 10^{-6} \mathrm{~mol} \mathrm{~L}^{-1}$ de selenito sobre m-AgSAE empregando-se potencial de preconcentração $0 \mathrm{~V}$ e t $=45 \mathrm{~s}$. (a) Empregando-se $f=100 \mathrm{~s}^{-1}, a=50 \mathrm{mV}$ e variando-se $\Delta \mathrm{E}_{\mathrm{s}}$. (b) Empregando-se $f=100 \mathrm{~s}^{-1}, \Delta \mathrm{E}_{\mathrm{s}}$ $=-2 \mathrm{mV}$ e variando-se $a$. (c) Empregando-se $\Delta \mathrm{E}_{\mathrm{s}}=-2 \mathrm{mV}, a=50 \mathrm{mV}$ e variando-se $f$.
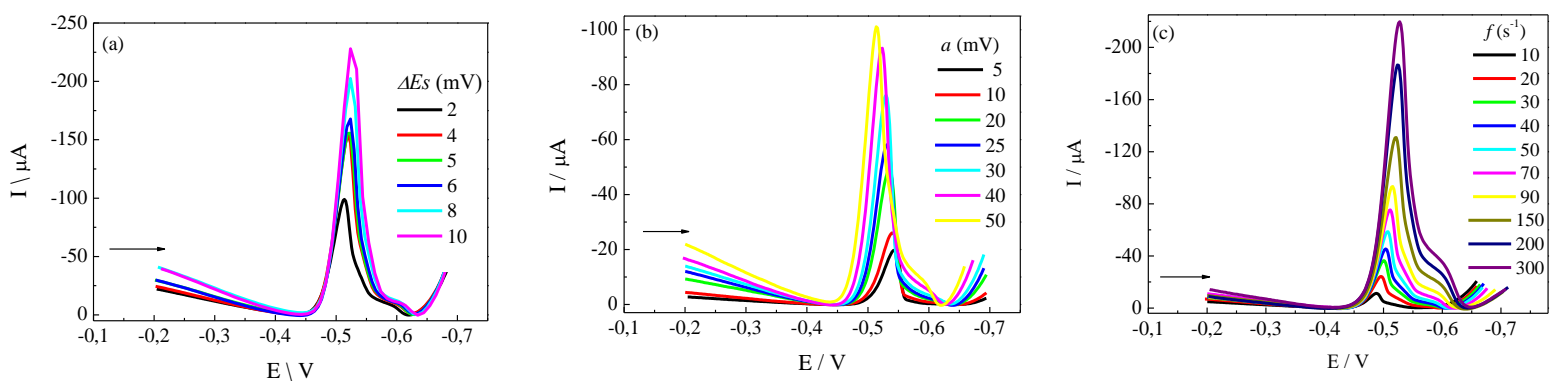

Assim, de acordo com os voltamogramas obtidos com a variação dos parâmetros voltamétricos foram definidos os seguintes valores: $a=25 \mathrm{mV}, \Delta \mathrm{E}_{\mathrm{s}}=-2 \mathrm{mV}$ e $f=90 \mathrm{~s}^{-1}$, os quais permitiram a construção de curvas analíticas com adições sucessivas de selenito à solução de eletrólito de suporte, na faixa de concentração de $1,00 \times 10^{-7}$ a $8,00 \times 10^{-6} \mathrm{~mol} \mathrm{~L}^{-1}$, como mostrado na Figura 3. 


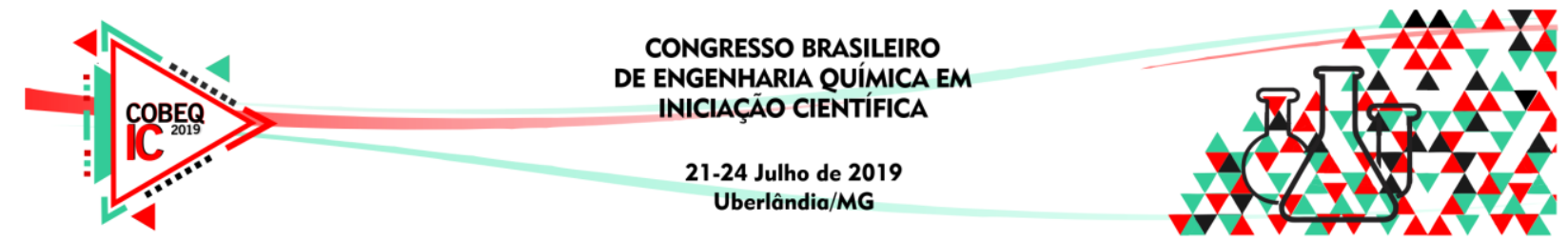

Figura 3 - (a) Voltamogramas de onda quadrada para a redução de $1,00 \times 10^{-6} \mathrm{~mol} \mathrm{~L}^{-1}$ de selenito sobre m-AgSAE empregando-se $f=90 \mathrm{~s}^{-1}, \Delta \mathrm{E}_{\mathrm{s}}=-2 \mathrm{mV}$ e $a=25 \mathrm{mV}$, potenciais de preconcentração $=0 \mathrm{~V}$ durante $45 \mathrm{~s}$. (b) Insert representa as relações de corrente de pico com concentração de selenito, obtidas a partir dos voltamogramas as curvas de calibração e a curva média.
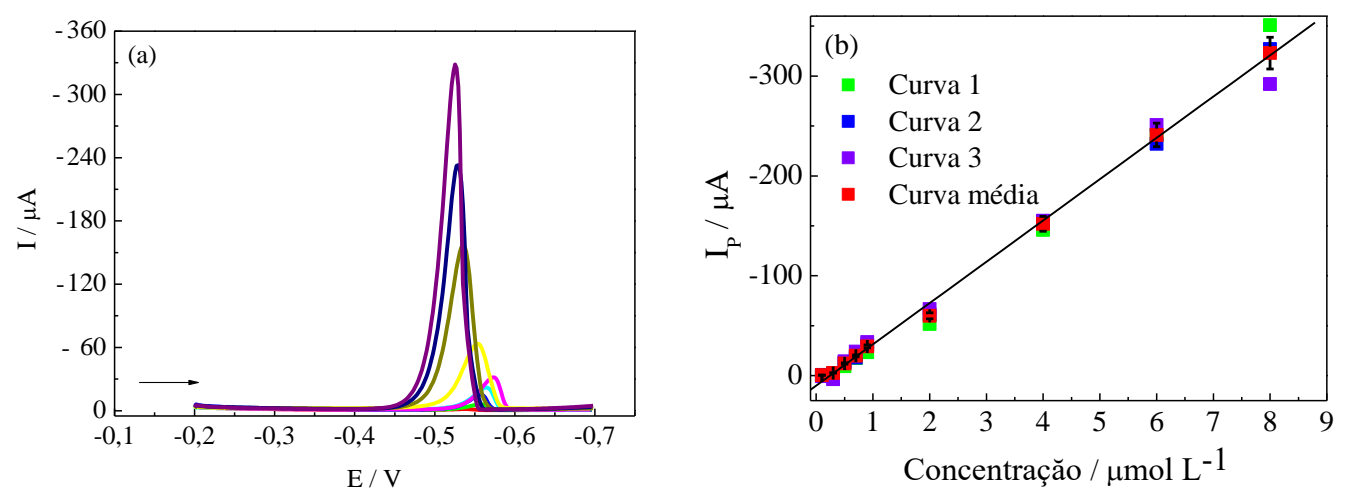

Os parâmetros analíticos foram avaliados e estão apresentados na Tabela 1 com comparação com métodos espectroscópicos empregando-se espectrometria de massa com plasma indutivamente acoplado (ICP-MS) e espectrometria de absorção atômica com forno de quartzo (QF-AAS).

Tabela 1 - Parâmetros analíticos para determinação analítica de selenito e comparação com dados da literatura.

\begin{tabular}{cccc}
\hline Parâmetros & Esse trabalho & ICP-MS [8] & QF-AAS [12] \\
\hline Faixa linear $\left(\mu \mathrm{g} \mathrm{L}^{-1}\right)$ & $0,10-8,0$ & $0,05-20$ & $0,4-100$ \\
Coeficiente de correlação $(\mathrm{r})$ & 0,9989 & 0,9986 & 0,9995 \\
Limite de detecção $\left(\mathrm{ng} \mathrm{L}^{-1}\right)$ & 2,9580 & 14,200 & 100,00 \\
Limite de quantificação $\left(\mathrm{ng} \mathrm{L}^{-1}\right)$ & 8,9636 & $\mathrm{NR}$ & $\mathrm{NR}$ \\
Recuperação (\%) & $101,00-101,10$ & $\mathrm{NR}$ & $\mathrm{NR}$ \\
Reprodutibilidade (\%) & 4,90 & 4,60 & 4,30 \\
Repetibilidade $(\%)$ & 1,64 & $\mathrm{NR}$ & $\mathrm{NR}$ \\
\hline
\end{tabular}

A recuperação empregando o eletrólito e o eletrólito digerido foi avaliado para a verificação da eficiência da técnica com uma concentração de $9 \times 10^{-7} \mathrm{~mol} \mathrm{~L}^{-1}$, foi obtido uma recuperação de 101,00 \% para o eletrólito digerido e 101,10\% para o eletrólito, o que demonstra que o eletrólito e o método de digestão não interferem nos sinais analíticos. De maneira análoga foi realizada a recuperação para as amostras previamente digeridas de maçã, laranja, batata, espinafre e tomate, e os valores calculados estão apresentados na Tabela 2. Desta maneira, observa-se que as eficiências de recuperações das amostras apresentaram valores maiores que aqueles obtidos no eletrólito, confirmando a presença de selenito nas amostras. Com estes resultados foi possível verificar que a metodologia desenvolvida e aplicada em alimentos in natura é eficiente para a determinação de selenito, sem interferência das diferentes composições das amostras empregadas. 


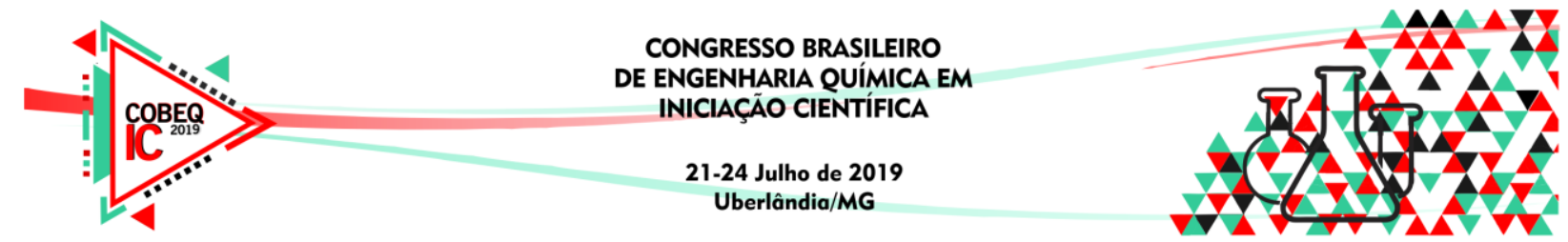

Tabela 2 - Concentrações encontradas, recuperações e BIAS para o selenito em amostras alimentícias.

\begin{tabular}{cccc}
\hline Amostra & Concentração encontrada $\left(\boldsymbol{\mu g} \mathbf{~ L}^{-\mathbf{1}}\right)$ & Recuperação $(\boldsymbol{\%})$ & BIAS (\%) \\
\hline Eletrólito & - & 101,10 & 1,10 \\
Eletrólito digerido & - & 101,00 & 1,00 \\
Maçã & 6,70 & 104,71 & 4,71 \\
Água de coco 1 & 27,52 & 115,49 & 15,49 \\
Água de coco 2 & 35,56 & 120,02 & 20,02 \\
Água de coco 3 & 43,38 & 124,42 & 24,42 \\
Laranja & 5,10 & 103,59 & 3,59 \\
Batata & 16,17 & 111,38 & 11,38 \\
Suco de soja 1 & 80,30 & 145,20 & 45,20 \\
Suco de soja 2 & 54,44 & 130,65 & 30,65 \\
Leite de soja 1 & 125,70 & 170,76 & 70,76 \\
Leite de soja 2 & 107,57 & 160,55 & 60,55 \\
Espinafre & 2,53 & 101,78 & 1,78 \\
Tomate & 38,45 & 127,05 & 27,05 \\
\hline
\end{tabular}

\section{CONCLUSÃO}

O emprego do m-AgSAE aliado à CS-SWV apresenta vantagens em função da diminuição de resíduos produzidos durante as análises, permite uma análise sensível, seletiva, reprodutiva e ainda rápida, sendo uma ótima técnica de identificação e quantificação de selenito. Além disso, esta metodologia pode ser comparada com resultados de metodologias espectroscópicas, e ainda foi possível a determinação de selenito em amostras de alimentos in natura.

\section{REFERÊNCIAS}

INAM, R.; SOMER, G.; Food Chem. v. 66, p. 381-389 (1999).

DE SOUZA, D. et al. Voltametria de onda quadrada. Segunda parte: aplicações. Química nova, v. 27, n. 5, p. 790-797, out./jan. 2004.

DE SOUZA, D.; MASCARO, L. H.; FATIBELO-FILHO, O. The effect of composition of solid silver amalgam electrodes on their electrochemical response. Journal of solid state electrochemistry, [S.L.], v. 15, n. 9, p. 2023-2029, nov. 2010.

MARTINS, F. C. O. L., et al. Compostos orgânicos e inorgânicos contendo selênio: revisão de métodos analíticos e perspectivas para análises químicas. Quím. Nova, v. 40, n.10, pp.1204-1214, 2017. 